\title{
Tradução e validação da versão brasileira da escala de gravidade na esclerose lateral amiotrófica (Egela)
}

\section{Translation and validation of the amyotrophic lateral sclerosis severity scale (ALSSS)}

\author{
Núbia Maria Freire Vieira Lima ${ }^{1}$, Celise Cirelli Guerra ${ }^{2}$, Luciane de Cássia Teixeira², \\ Luciano Bruno de Carvalho Silva ${ }^{3}$, Marina di Sordi ${ }^{4}$, Lúcia Mourão ${ }^{5}$, Anamarli Nucci ${ }^{6}$
}

Estudo desenvolvido no ambulatório de Doenças Neuromusculares do HC/ Unicamp - Hospital de Clínicas da Universidade Estadual de Campinas, Campinas, SP, Brasil

1 Profa. Ms. do Curso de Especialização em Neurologia Adulto da Ủnicamp

2 Fisioterapeutas especialistas em Fisioterapia Aplicada a Neurologia Adulto

3 Prof. Dr. do Depto. de Nutrição da Universidade Federal de Alfenas, Alfenas, MG

4 Fonoaudióloga, pesquisadora do Ambulatório de Disfagia do HC/UNICAMP

5 Profa. Dra. do Curso de Fonoaudiologia da FCM Faculdade de Ciências Médicas da Unicamp

6 Profa. Dra. associada do Depto. de Neurologia da FCM/ Unicamp

ENDEREÇO PARA CORRESPONDÊNCIA

Núbia M. F. V. Lima Depto. de Neurologia FCM Unicamp

Caixa postal 6111

13083-970 Campinas SP e-mail: nubia@fcm.unicamp.br

Estudo desenvolvido com apoio da Capes - Coordenação de Aperfeiçoamento de Pessoal de Nível Superior

APRESENTAÇÃO

abr. 2009

ACEITO PARA PUBLICAÇÃO

out. 2009
Resumo: O objetivo do trabalho foi traduzir a Amyotrophic Lateral Sclerosis Severity Scale para o português, como Escala de gravidade da esclerose lateral amiotrófica (Egela), além de validar e estudar sua confiabilidade. A escala foi submetida à versão e retroversão por tradutores bilíngües e três fisioterapeutas treinaram para padronizar sua aplicação. Foram avaliados 22 pacientes (5 mulheres, 17 homens, média de idade 45,9 anos) pela Egela e pela medida de independência funcional (MIF); 11 foram examinados para classificação de disfagia. Os coeficientes de correlação intraclasse dos domínios da Egela foram acima de 0,89. Foi constatada alta consistência interna em todos os seus domínios e para cada avaliador; foram encontradas fortes correlações entre a MIF motora e o escore espinhal da Egela $(\mathrm{r}=0.87$ e $p<0,0001)$, o domínio deglutição da Egela com as classificações de disfagia $(\mathrm{r}=-0.88$ e $p=0.0015)$, e o domínio fala da Egela com MIF expressão $(r=0,76$ e $p<0.001)$. A Egela mostrou significativa confiabilidade inter-examinador e consistência interna, além de correlação com os escores da escala MIF e de disfagia, permitindo sua validação e confiabilidade como instrumento de avaliação fucional de pacientes com esclerose lateral amiotrófica.

Descritores: Avaliação da deficiência; Esclerose amiotrófica lateral; Reprodutibilidade dos testes

ABSTRACT: The purpose was to translate the Amyotrophic Lateral Sclerosis Severity Scale (ALSSS) into Portuguese, to validate it and assess its reliability. The scale was submitted to bilingual translators, its abbreviation in Portuguese being Egela; three physical therapists were trained for its application. Twenty-two patients ( 5 women, 17 men, mean age 45.9) were evaluated by the Egela and by the functional independence measure (FIM); 11 of them were assessed for dysphagia classification. In all ALSSS domains the intraclass correlation coefficients were over 0.89; high internal consistency was found in all scale domains and for each examiner. Strong correlations were found between motor FIM and spinal ALSSS $(r=0.87 ; p<0.0001)$, ALSSS swallow domain and both dysphagia classifications $(r=-0.88 ; p=0.0015)$, and ALSSS speech domain and expression FIM $(r=0.76 ; p<0.001)$. The Portuguese version of ALSSS showed significant inter-examiner reliability and internal consistency, as well as strong correlations with FIM scores, thus proving a valid and reliable tool for assessing patients with amyotrophic lateral sclerosis.

Key words: Amyotrophic lateral sclerosis; Disability evaluation; Reproducibility of results 


\section{INTRODUÇÃO}

A esclerose lateral amiotrófica (ELA) é doença degenerativa que se manifesta por paralisia e atrofia muscular. No Brasil, a prevalência estimada varia de 0,9 a 1,5 casos/100.000 habitantes e a incidência, de 0,2 a 0,32 casos /100.000 habitantes/ano ${ }^{1}$. Os homens são afetados mais freqüentemente que mulheres, na proporção de um para dois ${ }^{2}$; e foi encontrada a incidência de 1:1,8 em série nacional $^{3}$.

A paralisia decorrente da ELA pode iniciar-se em membros ou orofaringe. Com freqüência, as mãos são afetadas primeiramente e cãibras musculares são sintomas característicos no início da doença ${ }^{4}$. A paralisia se torna mais grave, e extensiva a outras regiões do corpo, ocasionando crescente estado de dependência para cuidados pessoais, locomoção e alimentação, até o confinamento ao leito, entre 2 e 4 anos $^{5}$. A ingestão calórica inadequada e perda de peso corporal associadas à disfagia média a grave podem levar à desnutrição e desidratação $\mathrm{O}^{6,7}$.

Ventilação mecânica com pressão positiva não-invasiva tem sido usada com freqüência crescente na $\mathrm{ELA}^{8,9}$ para meIhorar a dispnéia noturna, insônia e desconforto respiratório e pode prolongar a vida. Entretanto, a evolução da doença pode exigir a indicação de ventilação mecânica invasiva. Uma vez efetuada a traqueostomia, o paciente pode ser mantido vivo durante anos, ainda que inteiramente paralisado. A morte do paciente com ELA decorre de insuficiência respiratória, pneumonia por aspiração ou embolia pulmonar após imobilidade prolongada ${ }^{10}$. A sobrevida média é de 4 a 5 anos e 20\% dos pacientes vivem mais de 5 anos.

Até o momento, não há tratamento curativo e, em conseqüência, o paciente requer assistência quanto à mobilidade e atividades da vida diária, atenção de diversos profissionais de saúde e ventilação mecânica ${ }^{10-12}$. A progressão inexorável da ELA exige que os profissionais proponham metas terapêuticas diversas, as quais diferirão de acordo com seu estado funcional. Torna-se imprescindível identificar e documentar as disfunções existentes, de modo reprodutível ${ }^{13,14}$, por meio de escalas funcionais.
É postulado que as escalas funcionais são de fácil aplicação, sem custos e não requerem equipamento especial. Pesquisadores apontam que medidas quantitativas precisas são necessárias para definir o distúrbio neuromuscular, documentar os dados terapêuticos, permitir mudanças em planos assistenciais e definir o estado natural da doença. Segundo Jackson ${ }^{14}$, selecionar corretamente o instrumento de medida na doença neuromuscular é a mais importante tarefa para o pesquisador que deseja entender o tratamento dessa condição. Entre os instrumentos disponíveis tem-se a Escala de Norris ${ }^{15}$, a Amyotrophic Lateral Sclerosis Severity Scale (ALSSS) ${ }^{16}$, a Amyotrophic Lateral Sclerosis Rating Functional Scale (ALSRFS-R) ${ }^{17}$. Contudo, nenhuma delas foi traduzida para a língua portuguesa ou validada no Brasil.

A ALSSS, ou escala de gravidade de ELA (Egela), foi criada por Hillel et al. ${ }^{16}$ em 1989 e avalia a evolução clínica e funcional da doença. Hillel et al. mensuraram sua confiabilidade, relações entre suas dimensões e relações com o óbito do paciente. A Egela foi utilizada na avaliação de disfagia em pacientes japoneses com ELA ${ }^{18,19}$. A escala contempla a função motora de extremidades superiores e inferiores durante as atividades de vida diária, bem como a fala e deglutição, o que a torna relevante para a avaliação multidisciplinar.

O estudo objetivou traduzir a ALSSS para o português - resultando na Egela -, analisar sua confiabilidade e efetuar a validação, por meio da correlação com a medida de independência funcional (MIF) e duas escalas de disfagia.

\section{METODOLOGIA}

Este estudo foi desenvolvido no Ambulatório de Doenças Neuromusculares do HC da Unicamp, aprovado pelo Comitê de Ética e Pesquisa da instituição, com assinatura do termo de consentimento livre e esclarecido pelos pacientes.

Foram incluídos pacientes entre $20 \mathrm{e}$ 70 anos, com diagnóstico clínico definido de ELA pelo critério El Escorial ${ }^{20}$, na forma bulbar, espinhal ou mista, usando ou não dispositivos auxiliares de loco- moção ou alimentação. Os indivíduos deveriam estar em uso do medicamento Riluzole. Os critérios de exclusão adotados foram: uso de ventilação mecânica invasiva, dificuldade na compreensão de instruções simples ou comorbidades neurológicas. Foram selecionados 22 pacientes.

A avaliação dos pacientes consistiu na aplicação da escala de gravidade de ELA, da medida de independência funcional (MIF) e de avaliação clínica da deglutição.

A Egela - escala de gravidade de ELA ${ }^{16}$ - é uma escala ordinal com quatro dimensões: extremidade inferior (EI), extremidade superior (ES), fala (F) e deglutição (D). Cada dimensão é pontuada pelo examinador de 10 a 1 , seguindo o declínio da função, adaptações funcionais, dispositivos auxiliares e necessidade de cuidador. A pontuação total varia de 40 (normal) a 4 (pior função) e não há escore classificatório de comprometimento pela Egela. O escore espinhal inclui as dimensões El e ES e o escore bulbar é a soma dos escores $F$ e D (Quadro 1 anexo).

A MIF contém seis domínios (autocuidado, controle dos esfíncteres, mobilidade, locomoção, comunicação e cognição social), com pontuação total de 126 (independência plena). Foram consideradas as dimensões motora, cognitiva e total e os subitens locomoção, cuidados pessoais e expressão ${ }^{21}$. A MIF foi utilizada como instrumento para validação construtiva da Egela.

A avaliação clínica da deglutição foi feita por fonoaudiólogo que participou do processo de tradução da Egela. Foram usadas as consistências líquida, líquidoengrossada (mel e néctar), pastosa (pudim) e sólida nas quantidades 3, 5 e 10 $\mathrm{ml}$. A gravidade da disfagia foi classificada de acordo com Furkim e Silva ${ }^{22}$ (escore 0, ausência de disfagia; 1, disfagia leve; 2, moderada; e 3, grave), seguindo a classificação clínica de disfagia de Yorkston et al. ${ }^{23}$.

Um teste de função pulmonar, no Laboratório de Prova de Função Pulmonar, foi aplicado em 11 participantes, sendo considerada a porcentagem de capacidade vital forçada (CVF\%). 


\section{Procedimentos}

Tradução: o instrumento original ${ }^{16}$ fol traduzido por dois tradutores bilíngües independentes que tinham como língua nativa o português; foram obtidas duas versões diferentes do questionário que foram analisadas por comitê de especialistas - um fisioterapeuta, um nutricionista e um fonoaudiólogo bilíngües - que definiram a versão em língua portuguesa. Esta última foi submetida à retroversão por um terceiro tradutor que não conhecia o instrumento original. A versão original, a versão em português e a retroversão foram comparadas pelo comitê de especialistas e eliminados os conflitos culturais ou de interpretação. A tradução e análise da confiabilidade no Brasil foram permitidas pelos autores da escala.

Treinamento dos examinadores e préteste: três fisioterapeutas participaram de treinamento teórico-prático para instruções e padronização da aplicação da escala. O pré-teste consistiu na aplicação do instrumento em três indivíduos com o diagnóstico de ELA, que não foram incluídos entre os 22 estudados. Após o pré-teste, itens da dimensão deglutição sofreram ajustes.

Avaliação: Os pacientes foram avaliados por uma equipe multidisciplinar. A MIF foi aplicada por um fisioterapeuta; a classificação da disfagia, feita por um fonoaudiólogo; e o estado nutricional, avaliado por nutricionista. Em seguida, os pacientes foram encaminhados a uma sala e individualmente submetidos à Egela, simultaneamente pelos três examinadores. Apenas 11 pacientes foram submetidos ao teste de função pulmonar e à avaliação da deglutição, pois houve casos de forma bulbar que impediam as provas pulmonares, somados ao nãocomparecimento de alguns pacientes para avaliação de deglutição. As avaliações ocorreram na mesma semana.

Para determinação do escore na Egela, os avaliadores procederam a rápido exame físico, sendo avaliada a necessidade de auxílio de alguém ou dispositivo auxiliar, ou recorreram a relato do paciente - ou de familiar, nos casos de disartria grave. Para pontuação da dimensão El (extremidade inferior), solicitaram ao paciente que caminhasse três metros, retornando à postura sentada. Os examinadores compararam a tarefa desempenhada ao relato do paciente/familiar, ou seja, se o paciente caminhou durante o exame sem assistência (escore 7 ou mais); mas se o familiar relatasse que o paciente apresentou quedas freqüentes, seria dado o escore de caminhada com ajuda (escore 6 ou menos), como sugerido por Hillel et al. ${ }^{16}$. Não foi estipulado tempo máximo para realização da atividade.

\section{Análise estatística}

As variáveis numéricas e categóricas foram submetidas à análise descritiva. A idade foi distribuída nas faixas $<40$ anos; $40-50$ anos; e $=50$ anos; o tempo de sintomas, em menor ou maior que 24 meses. A consistência interna foi avaliada pelo alfa de Cronbach, cujos valores acima de 0,70 indicam alta consistência interna ${ }^{24}$. Para averiguar correlações entre os vários instrumentos usou-se o coeficiente de correlação de Pearson (r). A confiabilidade inter-observador foi verificada nos quatro domínios da Egela e em sua pontuação total usando-se o coeficiente de correlação intraclasse (CCl): $\mathrm{CCl}$ $<0,40$ - concordância fraca; $\mathrm{CCl}<0,75$ - concordância moderada; e CCI >0,75 -alta concordância ${ }^{25}$. A confiabilidade inter-examinador foi verificada pela análise de Bland-Altman. O nível de significância adotado foi de 5\%. Foi utilizado o programa Statistical Analysis System.

\section{RESULTADOS}

Os dados demográficos da amostra estão resumidos na Tabela 1. A maioria eram homens, na faixa dos 40 anos. A Tabela também apresenta os escores médios obtidos na MIF e nas classificações da disfagia (nesse caso, referentes a apenas 11 pacientes).
A Tabela 2 apresenta os resultados da concordância entre os três avaliadores. O Gráfico 1 ilustra a boa concordância interexaminador entre o primeiro e terceiro avaliadores (A1 e A3).

Foi encontrada correlação moderada entre os escores de El e ES ( $r=0,66$ e $p<0,0007)$ e entre os escores de $\mathrm{D}$ e $\mathrm{F}$ $(r=0,53$ e $p<0,01)$. Os coeficientes de consistência interna para os domínios espinhal, bulbar e escore total foram $0,79,0,82$ e 0,64 , respectivamente. Não foi detectada correlação entre o escore espinhal e o escore bulbar $(r=0,234$; $p=0,29$ ). Nos 11 pacientes submetidos à avaliação clínica de deglutição encontrou-se correlação entre o escore de deglutição da Egela (D-Egela) e a classificação de Furkim e Silva ( $r=-0.88$; $p=0,0015)$; e entre a D-Egela e a classificação de Yorkston $(r=-0.88$; $p=0,0015$ ).

Também foi encontrada correlação moderada significante entre a capacidade vital forçada e a Egela total $(r=0,68$; $p<0,0001)$, mas não houve correlação 
Tabela 2 Concordância da Egela entre os três avaliadores

\begin{tabular}{lccc}
\hline Dimensões & $\mathrm{CCl}$ & $p$ & Int 95\% \\
\hline Extremidade inferior & 0,910 & $p<0,001$ & 0,$827 ; 0,958$ \\
Extremidade superior & 0,895 & $p<0,001$ & 0,$801 ; 0,951$ \\
Fala & 0,913 & $p<0,001$ & 0,$833 ; 0,96$ \\
Deglutição & 0,981 & $p<0,001$ & 0,$962 ; 0,922$ \\
Escore espinhal & 0,946 & $p<0,001$ & 0,$894 ; 0,975$ \\
Escore bulbar & 0,966 & $p<0,001$ & 0,$932 ; 0,984$ \\
Escore total & 0,968 & $p<0,001$ & 0,$935 ; 0,985$ \\
\hline
\end{tabular}

$\mathrm{CCl}=$ coeficiente de correlação intraclasse; Int 95\% = intervalo de $95 \%$ de confiança do CCl

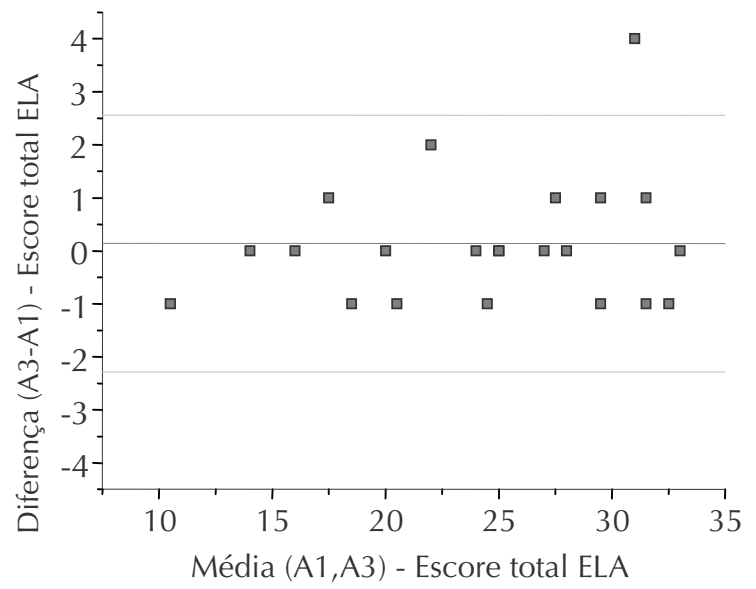

Gráfico 1 Concordância entre avaliadores (A1, A3) quanto aos escores totais na ELA atribuídos aos pacientes

com significância estatística entre a CVF\% e o escore bulbar da Egela, nem entre o escore de deglutição e a CVF\%. Houve forte correlação entre a CVF\% e a dimensão extremidade inferior da Egela $(r=0,82 ; p<0,003)$, mas não entre a CVF\% e a extremidade superior $(r=0,38 ; p=0,17)$.

Quanto às relações entre os escores nas dimensões da Egela e dos domínios da MIF, foram encontradas fortes correlações entre El e locomoção ( $r=0,9$; $p<0,001)$; entre ES e cuidados pessoais $(r=0,81 ; p<0,0001)$; entre fala e expressão $(r=0,76 ; p<0,001)$; entre escore espinhal e domínio motor $(r=0,87$ e $p<0,0001)$; e, ainda, entre o escore bulbar da Egela a o domínio cognitivo da MIF $(r=0,75$ e $p<0,0001)$.

\section{DISCUSSÃO E CONCLUSÃO}

A Egela, criada por Hillel et al. ${ }^{16}$ para avaliar a evolução clínica e funcional da doença, é considerada de fácil e rápida aplicação e foi validada nos EUA quanto à confiabilidade, relações entre suas dimensões e relações com o óbito do paciente ${ }^{16}$. Engloba atividades diárias que implicam redução da habilidade motora ou até mesmo necessidade de auxílio parcial ou total. Dessa forma, o uso seqüencial da escala pode mensurar a função em estudos longitudinais terapêuticos. Hillel et al. ${ }^{16}$ acompanharam 14 portadores de ELA ao longo de 4 a 14 meses, com média de tempo de sintomas de 8,6 meses na primeira avaliação. O grupo mostrou em média progressão na Egela de -11,3 pontos/ano e uma variação de $-3,4$ a -24 pontos/ ano $^{16}$.

A Egela mostrou excelente confiabilidade interexaminador na avaliação clínica da ELA e correlação significativa com os escores da MIF e escores de disfagia, além de apresentar boa consistência interna. Hillel et al. ${ }^{16}$ encontraram correlação interexaminadores com valores de $\mathrm{CCl}$ de 0,95 para El; 0,93 para
ES; 0,99 para F; e 0,95 para D, similares aos do presente estudo (Tabela 2). Neste estudo não foi realizado reteste: a avaliação dos pacientes em ambulatório e sua dependência de um cuidador para deslocamentos foram fatores que impediram sua realização.

A consistência interna da Egela não foi estudada em pesquisas anteriores. Os escores bulbar e espinhal mostraram excelente consistência interna. A dimensão D-Egela tem sido usada internacionalmente ${ }^{18,19}$. No estudo, a excelente correlação da D-Egela com os escores de disfagia, medida por dois outros instrumentos ${ }^{22,23}$, reforça a utilidade da escala nesse domínio.

A forte correlação da Egela com a MIF sugere que a primeira pode ser considerada instrumento de avaliação da independência funcional, ambas avaliando a presença do cuidador nas tarefas do paciente. De Groot et al. ${ }^{26}$ avaliaram pacientes de ELA com quatro escalas de atividades funcionais e apontaram a MIF como o instrumento mais adequado para mensuração do desempenho motor na ELA. A MIF oferece sete possibilidades de pontuação, ao passo que a Egela permite dez variações de função para cada dimensão. Orsini et al. ${ }^{27}$ destacam que a sensibilidade é uma propriedade psicométrica de grande importância para escalas funcionais. A Egela é o instrumento especifico para pacientes com ELA que apresenta o maior número de possibilidades de escore para cada domínio (dez), o que pode interferir na detecção de sinais ou sintomas anormais durante o exame físico e, assim, na meIhor discriminação dos pacientes.

Entre as limitações da Egela sublinhase a ausência da contemplação da função respiratória. Hillel encontrou uma fraca correlação entre a dimensão D da Egela e a CVF\%, tal como no presente estudo. É fato que os pacientes com comprometimento bulbar comumente apresentam maior dificuldade em efetuar o teste de função pulmonar e, por isso, não foi possível avaliar a CVF de todos os pacientes. O tempo de aplicação da Egela, de cerca de 10 minutos em média, torna-a viável tanto para o paciente quanto para o profissional. A forma original da Egela não apresenta manual de 
aplicação, o que não comprometeu o entendimento e aplicação do instrumento. Contudo, os aspectos das funções de extremidades inferiores e superiores podem ser mais detalhados e, futura- mente, implicar a inclusão de explicações que facilitem sua aplicação.

A Egela mostrou significativa confiabilidade interexaminador e consistência interna, além de correlação com os esco- res da escala MIF e de disfagia, o que permite afirmar sua validação e confiabilidade como instrumento na avaliação da esclerose lateral amiotrófica na prática ambulatorial ou hospitalar.

\section{REFERENNCIAS}

1 Dietrich-Neto F, Callegaro D, Dias-Tosta E, Silva HA, Ferraz ME, Lima JMB, et al. Amyotrophic lateral sclerosis in Brazil: 1998 national survey. Arq Neuropsiquiatr. 2000;58(3A):607-15.

2 Mitchell JD. Amyotrophic lateral sclerosis: toxins and environment. Amyotroph Lateral Scler Other Motor Neuron Disord. 2001;1:235-50.

3 Castro-Costa CM, Oriá RB, Vale OC, Arruda JAM, Horta WG, D'Almeida JAC, et al. Motor neuron diseases in the University Hospital of Fortaleza (Northeastern Brazil). Arq Neuropsiquiatr. 2000;58(4):986-9.

4 França MC Jr, D'Abreu A, Nucci A, Lopes-Cendes I. Muscle excitability abnormalities in Machado-Joseph disease. Arch Neurol. 2008;65(4):525-9.

5 Borasio GD. Palliative care in ALS. J Neurol. 1997;244:s11-s17.

6 Kasarskis E, Berryman S, Vanderleest JG, Schneider AR, McClain CJ. Nutritional status of patients with amyotrophic lateral sclerosis: relation to the proximity of death. Am J Clin Nutr. 1996;63(1):130-7.

7 Silva LBC, Mourão LF, Silva AA, Lima NMFV, Franca Junior M, Nucci A, et al. Avaliação da ingestão alimentar de indivíduos com esclerose lateral amiotrófica. Rev Bras Nutr Clin. 2008;23(1):5-12.

8 Jackson CE, Rosenfeld J, Moore DH, Bryan WW, Barohn RJ, Wrench $\mathrm{M}$, et al. A preliminary evaluation of a prospective study of pulmonary function studies and symptoms of hypoventilation in ALSrMND patients. J Neurol Sci. 2001;191:75-8.

9 Janssens JP, Derivaz S, Breitenstein E, De Muralt B, Fitting JW, Chevrolet JC, et al. Changing patterns in long-term noninvasive ventilation: a 7 -year prospective study in the Geneva Lake area. Chest. 2003;123:67-79.

10 Beghi E, Logroscino G, Chiò A, Hardiman O, Mitchell $D$, Swingler R, et al. The epidemiology of ALS and the role of population-based registries. Bioch Biophy Acta. 2006;1762: 1150-7.

11 Borasio GD, Voltz R, Miller RG. Palliative care in amyotrophic lateral sclerosis. Neurol Clin. 2001;19:829-47.

12 Mitsumoto H. Diagnosis and progression of ALS. Neurology. 1997;48:S2-S8.

13 Andres PL, Skerry LM, Thornell B, Portney LG, Finison LJ, Munsat TL. A comparison of three measures of disease progression in ALS. J Neurol Sci. 1996;139:64-70.
14 Jackson CE, Rosenfeld J. Quantitative assessment and outcome measures in neuromuscular disease. In: Katirji B, Kaminski HJ, Preston DC, Ruff RL, Shapiro BE. Neuromuscular disorders in clinical practice. Ohio: Butterworth Heinemann; 2002. p.309-38.

15 Norris FH. Administration of guanidine in ALS. Neurology. 1974;24:728-?.

16 Hillel AD, Miller RM, Yorkston K, McDonald E, Norris FH, Konikow N. Amyotrophic lateral sclerosis severity scale. Neuroepidemiology. 1989;8:142-50.

17 Cedarbaum JM, Stambler N, Malta E, Fuller C, Hilt D, Thurmond B, et al. The ALSFRS-R: a revised ALS functional rating scale that incorporates assessments of respiratory function. J Neurol Sci. 1999;169:13-21.

18 Kawai S, Tsukuda M, Mochimatsu I, Enomoto H, Kagesato Y, Hirose $\mathrm{H}$, et al. A study of the early stage of dysphagia in amyotrophic lateral sclerosis. Dysphagia. 2003;18(1):1-8.

19 Higo R, Tayama N, Nito T. Longitudinal analysis of progression of dysphagia in amyotrophic lateral sclerosis. Auris Nasus Larynx. 2004;31:247-54.

20 Brooks BR, Miller RG, Swash M, Munsat TL. World Federation of Neurology Research Group on Motor Neuron Diseases, El Escorial revisited: revised criteria for the diagnosis of amyotrophic lateral sclerosis. Amyotroph Lateral Scler Other Mot Neuron Disord. 2000;1:293-9.

21 Riberto M, Miyazaki MH, Jucá SSH, Sakamoto H, Pinto PPN, Batistella LR. Validação da versão brasileira da medida de independência funcional. Acta Fisiatr. 2004;2:72-6.

22 Furkim AM, Silva RG. Programas de reabilitação em disfagia neurogênica. São Paulo: Frôntis; 1999.

23 Yorkston KM, Miller RM, Strand EA. Management of speech and swallowing in degenerative diseases. Austin: Pro-Ed; 2004.

24 Nunnally JC. Psychometric theory. New York: McGrawHill; 1978.

25 Fleiss JL. Statistical methods for rates and proportions. 2nd ed. New York: John Wiley \& Sons; 1981.

26 De Groot IJM, Post MWM, Post MW, Van Heuveln T, Van Den Berg LH, Lindeman E. Measurement of decline of functioning in persons with amyotrophic lateral sclerosis: responsiveness and possible applications of the Functional Independence Measure, Barthel Index, Rehabilitation Activities Profile and Frenchay Activities Index. Amyotroph Lateral Scler Other Motor Neuron Disord. 2006;7:167-72.

27 Orsini M, Freitas MRG, Mello MP, Botelho JP, Cardoso FM, Nascimento OJM, et al. Medidas de avaliação na esclerose lateral amiotrófica. Rev Neurocienc. 2008;16(2):144-51. 
Quadro 1 Escala de gravidade de ELA: extremidades superiores, extremidades inferiores, fala e deglutição

EXTREMIDADES INFERIORES (CAMINHAR)

\section{Normal}

10 Marcha sem alterações

9 Suspeita de fadiga

Início de dificuldade na marcha

8 Dificuldade em terreno desnivelado

7 Mudanças observadas na marcha

Caminhada com ajuda

6 Caminhada com dispositivo mecânico

5 Caminhada com dispositivo mecânico e assistência (auxílio)

Somente movimento funcional

4 Capaz de suportar o próprio peso

3 Movimentos voluntários de membros inferiores

\section{Movimento da perna sem propósito}

O paciente nega qualquer fraqueza ou fadiga; exame não detecta anormalidade. O paciente refere fraqueza ou fadiga nos membros inferiores durante o esforço físico.

Dificuldade e fadiga ao caminhar longas distâncias, ao subir escadas e caminhar em chão irregular (ou carpete espesso).

Mudança notável na marcha; apoio em corrimão ao subir escada; pode usar órtese para a perna.

Necessita ou usa bengala, andador ou assistência para caminhar; pode usar cadeira de rodas para locomoção comunitária.

Não tenta caminhar sem ajuda de outra pessoa; caminhada limitada a

aproximadamente $1.525 \mathrm{~m}$; evita escadas.

No máximo, pode caminhar um pouco, arrastando os pés, com ajuda do cuidador para as transferências.

Incapaz de dar passos, mas pode posicionar as pernas para facilitar o trabalho do cuidador em transferências; move as pernas propositalmente para manter a mobilidade na cama.

2 Movimento mínimo

Movimento mínimo de uma ou ambas as pernas; não pode reposicionar as pernas independentemente.

1 Paralisia

Paralisia flácida; não pode mover os membros inferiores (exceto, talvez, em inspeção minuciosa).

EXTREMIDADES SUPERIORES (VESTIR-SE E FAZER HIGIENE PESSOAL)

\section{Normal}

10 Função sem alterações

9 Suspeita de fadiga

\section{Auto-cuidado independente e completo}

8 Auto-cuidado lento

7 Desempenho de auto-cuidado com esforço

\section{Assistência intermitente}

Independência na maior parte do tempo

5 Independência parcial
O paciente nega qualquer fraqueza ou fadiga incomum nos membros superiores; exame físico não demonstra anormalidade.

O paciente refere fadiga nos membros superiores durante esforço físico; não suporta trabalhar por períodos normais; atrofia não-evidente ao exame.

Veste-se e faz a higiene mais lentamente do que o normal

Requer maior tempo (geralmente o dobro ou mais) e esforço para concluir o autocuidado; fraqueza evidente ao exame.

Realiza muitas tarefas sozinho, como vestir-se e fazer sua higiene pessoal; adaptase descansando, modificando hábitos (exemplos) ou evitando algumas tarefas; requer assistência para pequenas atividades motoras (p. ex. abotoar, amarrar) Lida com algumas tarefas sozinho, como vestir-se e fazer a higiene pessoal, entretanto, precisa de assistência para muitas tarefas como: maquiar-se, pentear-se, barbear-se.

Necessidade de ajuda para cuidados pessoais

4 Assistência do cuidador

O cuidador deve estar presente para atividades tais como: vestir-se e fazer higiene pessoal; o paciente realiza a maioria das tarefas com a ajuda do cuidador.

3 Paciente auxilia o cuidador

Dependência total

2 Movimento mínimo

1 Paralisia
O cuidador conduz o paciente para quase todas as tarefas; o paciente se move de modo proposital para ajudar o cuidador; o paciente não inicia o auto-cuidado.

Movimento mínimo de um ou ambos os braços; incapaz de reposicionar os braços. Paralisia flácida; incapaz de mover os membros superiores (exceto, talvez, em inspeção minuciosa). 
FALA

\section{Normal}

10 Fala sem alterações

9 Anormalidades na fala

\section{Distúrbio detectável no discurso}

8 Mudanças perceptíveis na fala

7 Anormalidades evidentes na fala

\section{Compreensível com repetição}

6 Repetição ocasional da

mensagem

5 Repetição solicitada com

freqüência

\section{comunicação não-verbal}

4 Fala e comunicação não-verbal

3 Fala limitada a respostas monossilábicas

Perda do discurso útil

2 Vocalização para expressão

emocional

1 Não-sonoro
Paciente nega qualquer dificuldade na fala; o exame não detecta anormalidade.

Somente o paciente ou o cônjuge nota que a fala mudou; mantém a velocidade e o volume normais.

Mudanças no discurso são notadas por outras pessoas, principalmente em situação de fadiga ou estresse; a velocidade da fala permanece essencialmente normal.

A fala é consistentemente deficiente; a velocidade, a articulação e a ressonância são afetadas; permanece compreensível.

A velocidade é muito mais lenta, repete palavras específicas em ambientes com ruídos adversos; não limita a complexidade ou a extensão da mensagem.

A fala é lenta e trabalhosa; repetição ou "tradução" são geralmente necessárias, o paciente limita a complexidade ou extensão da mensagem.

A fala é utilizada em resposta a perguntas; mensagens incompreensíveis precisam ser resolvidas por escrito ou por meio de intérprete.

Vocaliza respostas de uma palavra, por meio de sim / não, todavia escreve ou usa intérprete; inicia a comunicação não-verbal.

O paciente usa modulação vocal para expressar emoção, afirmação e negação.

A vocalização é feita com muito esforço, é limitada na duração e raramente é obtida; pode sonorizar para chorar ou expressar dor.

DEGLUTIÇÃO

\section{Hábitos alimentares normais}

10 Deglutição sem alterações

9 Queixa principal

\section{Problemas iniciais na alimentação}

8 Pequenos problemas na deglutição

Tempo de refeição aumentado/

7 ingestão de alimentos em pequenos pedaços

\section{Mudanças na consistência da dieta}

6 Dieta leve

A alimentação está limitada a alimentos macios; a refeição necessita de alguma preparação especial.

Ingestão oral adequada; nutrição limitada primeiramente para líquidos; ingestão de líquidos ralos geralmente é um problema; o paciente "se obriga" a comer.

5 Dieta líquida Necessidade de tubo para alimentação

4 Tubos alimentares suplementares

Via alternativa de nutrição com nutrição oral ocasional

O paciente nega qualquer dificuldade na mastigação ou deglutição; a avaliação não mostra alteração alguma.

Somente o paciente sente leves indicações, tais como discreto acúmulo de alimento na cavidade oral e na região laringo-faríngea.

O paciente queixa-se de alguma dificuldade na deglutição; mantém essencialmente uma dieta regular; episódios isolados de engasgos.

O tempo de refeição aumentou significativamente, e é necessário cortar pedaços menores de alimento; deve concentrar-se em engolir líquidos ralos.

Ingestão por via oral é insuficiente; paciente usa ou necessita de complementação nutricional por via alternativa; paciente ingere mais que $50 \%$ por via oral.

Maior parte da dieta administrada por via alternativa; recebe menos que $50 \%$ da dieta por via oral.

Nenhuma alimentação oral

2 Manejo das secreções com aspirador e/ou medicação

1 Aspiração de secreções
O paciente não pode realizar qualquer ingestão oral com segurança; no manejo das secreções usa aspirador e/ ou medicações; o paciente deglute reflexamente. As secreções são manipuladas de modo invasivo, exclusivo; o paciente deglute raramente. 